\title{
Electromagnetic Response Function for the Period of 27 Day in the Chinese Region
}

\author{
P. F. CHEN and P. C. W. Fung \\ Department of Physics, University of Hong Kong, Hong Kong
}

(Received March 28, 1991; Revised August 29, 1991)

\begin{abstract}
Using the "Sompi" spectral analysis method, the daily mean values for 14 geomagnetic observatories in the Chinese region have been analysed for determining the magnitudes and phases of the $P_{1}{ }^{0}$ response corresponding to the period of $27 \mathrm{~d}$. The contour maps for the apparent depth $h^{*}$ and the conductivity $\sigma$ of the perfect substitude conductor were then plotted for this period. The results indicate that the lateral changes of $\sigma$ and $h^{*}$ are not very large in the central and northern Chinese region, where the average value of $h^{*}$ is around $950 \mathrm{~km}$ and the average value of $\sigma$ is around $1.0 \mathrm{~S} / \mathrm{m}$. These values are basically in agreement with those obtained by SCHULTZ and LARSEN (1987) for the neighbouring observatories of China-Irkutsk, Memambetsu, Kakioka and Kanoya. In the southern Chinese observatories we have found lower values of $\sigma$ and larger values of $h^{*}$. It is not clear whether this characteristic feature in the southern Chinese region is due to the influence of the highly conducting ocean or the equatorial electrojet current or the poor signal to noise ratio in the data of $Z$ magnetic component.
\end{abstract}

\section{Introduction}

The previous works on the 27 day variation of the geomagnetic field have been reviewed in detail by ROBERTS (1986), and the corresponding response function can be used to study the deep conductivity structure in a global scale assuming that the Earth has a radially symmetric conductivity structure. It can also be used to study the structure in a regional scale because the magnetic variation field at the Earth's surface can be represented by zonal spherical harmonic $P_{1}{ }^{0}$. This assumption enables us to reveal the lateral inhomogeneity of the electrical conductivity, provided that the spatial changes in the conductivity are relatively gradual.

Based on the response at the 27 day period, PECOVA et al. (1980) discovered that in the European region the response function $W_{1}$ increases when the geomagnetic latitude decreases. This feature indicates that some deep E-W striking structures exist beneath the central European region. Recently, SCHULTZ and LARSEN (1987) show that the differences of the inductive scale lengths (such as that for the 27 day period) among 22 observatories, whose response functions are consistent with both the $P_{1}{ }^{0}$ assumption and local 1-D earth structure, are significant. Besides, ROBERTS (1984) also concluded that different responses were found at different locations and suggested that the influence of the highly conductive oceans should be taken into account in the calculation of the response functions. Nevertheless, the existence of 
the lateral inhomogeneity of electrical conductivity in mid-mantle is now widely accepted by geomagneticians.

We notice that the data of the Chinese observatories have never been used in the past literature. Therefore, the main purpose of this study is to examine the possible lateral change of the deep conductivity structure in the Chinese region. The second purpose is to examine the validity of the Sompi spectral method in the analysis of long period geomagnetic variations.

\section{Regional GDS Using the Single Station Data}

Assuming that the 27-day magnetic variation is solely caused by the ring current, which flows in the geomagnetic equator plane at a distance of several Earth radii, the magnetic field at the ground surface can be expanded in a series of odd order zonal spherical harmonics (BANKS, 1969):

$$
\begin{aligned}
& H(\theta, f)=\sum H_{n}(\theta, f)=\sum A_{H, n}(f) \frac{\partial P_{n}^{0}(\cos \theta)}{\partial \theta}, \\
& Z(\theta, f)=\sum Z_{n}(\theta, f)=\sum A_{Z, n}(f) P_{n}^{0}(\cos \theta) \\
& A_{H, n}(f)=i_{n}(f)+e_{n}(f), \\
& A_{Z, n}(f)=n e_{n}(f)-(n+1) i_{n}(f),
\end{aligned}
$$

where $H$ and $Z$ denote respectively the horizontal and the vertical components of the geomagnetic field, $\theta$ is the co-latitude in the geomagnetic coordinate system (i.e. in the dipole coordinate system), $f$ is the frequency, $i_{n}(f)$ and $e_{n}(f)$ represent respectively the internal and external parts of the field. The response function $W_{n}$ is defined as

$$
W_{n}=A_{Z, n}(f) / A_{H, n}(f) .
$$

In formula (1), $P_{1}{ }^{0}$ represents the main contribution to the series. The amplitude of the second term $P_{3}{ }^{0}$ is less than $10 \%$ of the amplitude of the first term $P_{1}{ }^{0}$. Therefore, the contribution of $P_{3}{ }^{0}$ and the other terms can be neglected in the following analysis (BANKS, 1969). In this case, from Eqs. (3) and (1) we have

$$
W_{1}=-\tan \theta[Z(\theta, f) / H(\theta, f)]
$$

The ratio of the internal part and the external part of the field is then determined by the following equation:

$$
Q_{1}(f)=i_{1}(f) / e_{1}(f)=\left(1-W_{1}\right) /\left(2+W_{1}\right)
$$

According to the perfect substitute conductor model suggested by SCHMUCKER (1970), the inductive scale length is (PARKINSON and HUTTON, 1989): 


$$
C=R\left(1-2 Q_{1}\right) / 2\left(1+Q_{1}\right)
$$

where $R$ is the radius of the Earth $(R=6370 \mathrm{~km})$. Since the substitute conductor model is constructed by a poorly conducting layer of thickness $d$ overlying a well-conducting half space of conductivity $\sigma$, the real part and the imaginary part of the inductive scale length becomes

$$
\begin{gathered}
C_{\text {real }}=d+\delta / 2=h^{*}, \\
C_{\text {imag }}=-\delta / 2,
\end{gathered}
$$

where $\delta$ is the skin depth of the lower conductive medium. Thus the real part of $C$ gives an estimate of the depth at which the induced currents flow. The imaginary part of $C$ can be used to calculate the conductivity $\sigma$ of the substitute conductor:

$$
\sigma=0.253 \cdot 86400 \cdot T / 4\left(C_{\text {imag }}\right)^{2}
$$

where $T$ is the inducing period of the field in unit of day, $\sigma$ is in unit of $\mathrm{S} / \mathrm{m}$.

\section{Sompi Spectral Analysis}

The Sompi method is a new and powerful spectral analysis technique. The theory of this method can be found in the literature of KUMAZAWA et al. (1990), ASAKAWA et al. (1988) and HORI et al. (1989). In such a method, one uses an autoregressive (AR) process model to analyze a stationary time series. The AR coefficients $\left\{a_{j}\right\}_{j=-m}^{m}$ are determined by solving the eigenvalue problem:

$$
\sum_{j=-m}^{m}\left(P_{j k}-\lambda \delta_{j k}\right) a_{j}=0 \quad(k=-m, \ldots, m)
$$

where $\delta_{j k}$ is the delta function, $2 m$ is specified as the AR order, and

$$
P_{j k}=P_{k j}=\frac{1}{2(N-m)+1} \sum_{i=-N+m}^{N-m} x_{i-j} x_{i-k} \quad(j, k=-m, \ldots, m)
$$

is the non-Toeplitz matrix, where $\left\{x_{n}\right\}_{n=-N}^{N}$ is the discrete time series. The eigenvector $\left\{a_{j}\right\}_{j=-m}^{m}$ corresponding to the minimum eigenvalue $\lambda_{\min }$ is the solution for the matrix in Eq. (10).

In this study, we shall analyze the spectra of the $H$ and $Z$ components of the geomagnetic data. We assume that the two components consist of common line spectra (i.e. the AR coefficients for the two components are the same). Besides, since the magnitude of the temporal $Z$ variation is smaller than that of the $H$ variation in the region of low and mid 
latitude, we give different weighting to the $H$ and $Z$ data for composing an appropriate nonToeplitz matrix:

$$
P_{j k}=P_{j k}^{H}+2 P_{j k}^{Z}
$$

where $P_{j k}{ }^{H}$ and $P_{j k}{ }^{Z}$ are respectively the autocovariance matrix of $H$ and $Z$ components of the geomagnetic variation.To facilitate quantitative analysis and to bring out the separate features of the $H$ and $Z$ variations, we need to assign a suitable numerical value for the weighting coefficient of $P_{j k}{ }^{Z}$. We find that the value of 2 is suitable for our analysis without lost of generality.

\section{Data Analysis}

The daily means of $H$ and $Z$ components for 14 permanent Chinese observatories were analysed based on the SCHMUCKER (1970) methodology to investigate the lateral variations of the conductivity $\sigma$ and the apparent depth $h^{*}$ of a perfect substitute conductor. The geomagnetic latitudes of the observatories and the years analysed are listed in Table 1.

The 256 data points in each year for each observatory, usually start from 1st January to September, are analysed by means of the Sompi spectral analysis. For comparison, the same data are also analysed using the FFT method.

To apply the Sompi method, the raw data are first preconditioned by removing the linear trend and passing through a band-pass filter. The band-pass filter used in this study is simply a box-car window in the frequency domain. A wide band-width of 0.027 to 0.195 in frequency domain is chosen for the analysis. After detrending and filtering, the time series are decimated in order to raise the $\mathrm{S} / \mathrm{N}$ ratio in the resultant spectrum. The sampling rate for the decimation procedure is chosen to be 3 in this study. The most difficult procedure in the Sompi method is how to select the optimum AR order. Here we use an empirical approach. When we scan the AR order in a certain range, we find that the maximum amplitudes of $H$ component and $Z$

Table 1. Geomagnetic latitudes of the observatories used in the analysis.

\begin{tabular}{lcl}
\hline \multicolumn{1}{c}{ Observatory } & $\begin{array}{c}\text { Geomagnetic } \\
\text { latitude } \\
\text { (degrees) }\end{array}$ & \multicolumn{1}{c}{ Years analysed } \\
\hline MZL (Manzhouli) & $38.5 \mathrm{~N}$ & 84,85 \\
URQ (Urumqi) & $33.3 \mathrm{~N}$ & $82,83,84$ \\
CHC (Changchun) & $33.2 \mathrm{~N}$ & $80,82,83,84$ \\
BJG (Beijing) & $28.9 \mathrm{~N}$ & $81,82,83,84$ \\
TAN (Tai-an) & $25.1 \mathrm{~N}$ & 84 \\
LZH (Lanzhou) & $24.9 \mathrm{~N}$ & $81,82,83,84$ \\
SHS (Sheshan) & $20.1 \mathrm{~N}$ & $80,81,82,83,84$ \\
CHD (Chengdu) & $19.8 \mathrm{~N}$ & $82,83,84,85$ \\
WUH (Wuhan) & $19.4 \mathrm{~N}$ & $81,82,83,84$ \\
LAS (Lhasa) & $18.9 \mathrm{~N}$ & 85 \\
QNZ (Quanzhou) & $13.9 \mathrm{~N}$ & 83 \\
TGH (Tonghai) & $13.0 \mathrm{~N}$ & 85 \\
GGZ (Guangzhou) & $11.9 \mathrm{~N}$ & $81,82,83,84$ \\
QGZ (Qiongzhong) & $7.8 \mathrm{~N}$ & 83,84 \\
\hline
\end{tabular}


component can be obtained for a searching frequency at a certain AR order. This order is treated as the optimum AR order and the corresponding amplitude and phase may be the optimum and the stable solution to the spectral analysis.

To apply the FFT method, the raw data are preconditioned by removing the linear trend and passing through the Hanning window.

Table 2 shows the comparison of the $C$ values of three observatories for the period near 25.6 days obtained by the Sompi method and the FFT method. The differences between the average values obtained by these two methods are not very large; however, the standard errors of the Sompi results are obviously smaller than that obtainable from the FFT results.

Figures 1 and 2 show respectively the distributions of the apparent depth $h^{*}$ and the conductivity $\sigma$ of the perfect substitute conductor. In Figs. 1 and 2 the values of $h^{*}$ and $\sigma$ for the Irkutsk (IRT), Memambetsu (MMB), Kakioka (KAK) and Kanoya (KNY) are taken from Table 2 in the article of SCHULTZ and LARSEN (1987). We can see that these values of $h^{*}$ and $\sigma$ compare reasonably well with those values from the neighbouring Chinese observatories determined in this study. The contour maps of $h^{*}$ and $\sigma$ show that the lateral change of the

Table 2. Comparison of the Sompi results and the FFT results for the Beijing, Wuhan and Lanzhou observatories.

\begin{tabular}{|c|c|c|c|c|}
\hline \multicolumn{5}{|c|}{ Beijing } \\
\hline Year & $C(\sim 25.6 \mathrm{~d})$ & [Sompi] & $C(25.6 \mathrm{~d})$ & {$[\mathrm{FFT}]$} \\
\hline 1981 & 1164.0 & $-437.3 i$ & 1042.9 & $-253.1 \mathrm{i}$ \\
\hline 1982 & 971.3 & $-97.6 \mathrm{i}$ & 821.8 & $44.6 \mathrm{i}$ \\
\hline 1983 & 1010.2 & $-336.7 i$ & 1271.2 & $63.5 \mathrm{i}$ \\
\hline 1984 & 1003.7 & $-627.7 \mathrm{i}$ & 1092.8 & $-732.6 \mathrm{i}$ \\
\hline $\begin{array}{c}\text { Average } \\
\text { Standard error }\end{array}$ & $\begin{array}{c}1037.3 \\
( \pm 86.2) \\
\end{array}$ & $\begin{array}{r}-374.8 \mathrm{i} \\
( \pm 220.7 \mathrm{i}) \\
\end{array}$ & $\begin{array}{r}1052.7 \\
( \pm 185.1) \\
\end{array}$ & $\begin{array}{r}-219.4 \mathrm{i} \\
( \pm 371.6 \mathrm{i}) \\
\end{array}$ \\
\hline \multicolumn{5}{|c|}{ Wuhan } \\
\hline Year & $C(\sim 25.6 \mathrm{~d})$ & [Sompi] & $C(25.6 \mathrm{~d})$ & [FFT] \\
\hline 1981 & 984.0 & $-480.8 \mathrm{i}$ & 1012.2 & $-503.3 \mathrm{i}$ \\
\hline 1982 & 640.8 & $-230.8 \mathrm{i}$ & 447.1 & $+476.9 \mathrm{i}$ \\
\hline 1983 & 1158.7 & $-84.5 \mathrm{i}$ & 1468.8 & $+67.1 \mathrm{i}$ \\
\hline 1984 & 932.9 & $-700.7 \mathrm{i}$ & 986.3 & $-755.4 \mathrm{i}$ \\
\hline $\begin{array}{c}\text { Average } \\
\text { Standard error }\end{array}$ & $\begin{array}{r}929.1 \\
( \pm 215.1) \\
\end{array}$ & $\begin{array}{r}-374.2 \mathrm{i} \\
( \pm 272.3 \mathrm{i}) \\
\end{array}$ & $\begin{array}{r}978.6 \\
( \pm 417.9) \\
\end{array}$ & $\begin{array}{r}-178.7 \mathrm{i} \\
( \pm 556.2 \mathrm{i}) \\
\end{array}$ \\
\hline
\end{tabular}

\begin{tabular}{ccccr}
\multicolumn{5}{c}{ Lanzhou } \\
\hline Year & $C(\sim 25.6 \mathrm{~d})$ & {$[$ Sompi] } & $C(25.6 \mathrm{~d})$ & {$[$ [FFT] } \\
\hline 1981 & 1234.4 & $-511.3 \mathrm{i}$ & 1209.4 & $-414.8 \mathrm{i}$ \\
1982 & 882.9 & $-208.4 \mathrm{i}$ & 769.5 & $-160.2 \mathrm{i}$ \\
1983 & 1084.8 & $-225.8 \mathrm{i}$ & 1398.5 & $-201.3 \mathrm{i}$ \\
1984 & 919.6 & $-573.0 \mathrm{i}$ & 890.7 & $-697.9 \mathrm{i}$ \\
& & & & \\
Average & 1030.4 & $-379.6 \mathrm{i}$ & 1067.0 & $-368.6 \mathrm{i}$ \\
Standard error & $( \pm 161.9)$ & $( \pm 189.5 \mathrm{i})$ & $( \pm 288.5)$ & $( \pm 246.3 \mathrm{i})$ \\
\hline
\end{tabular}




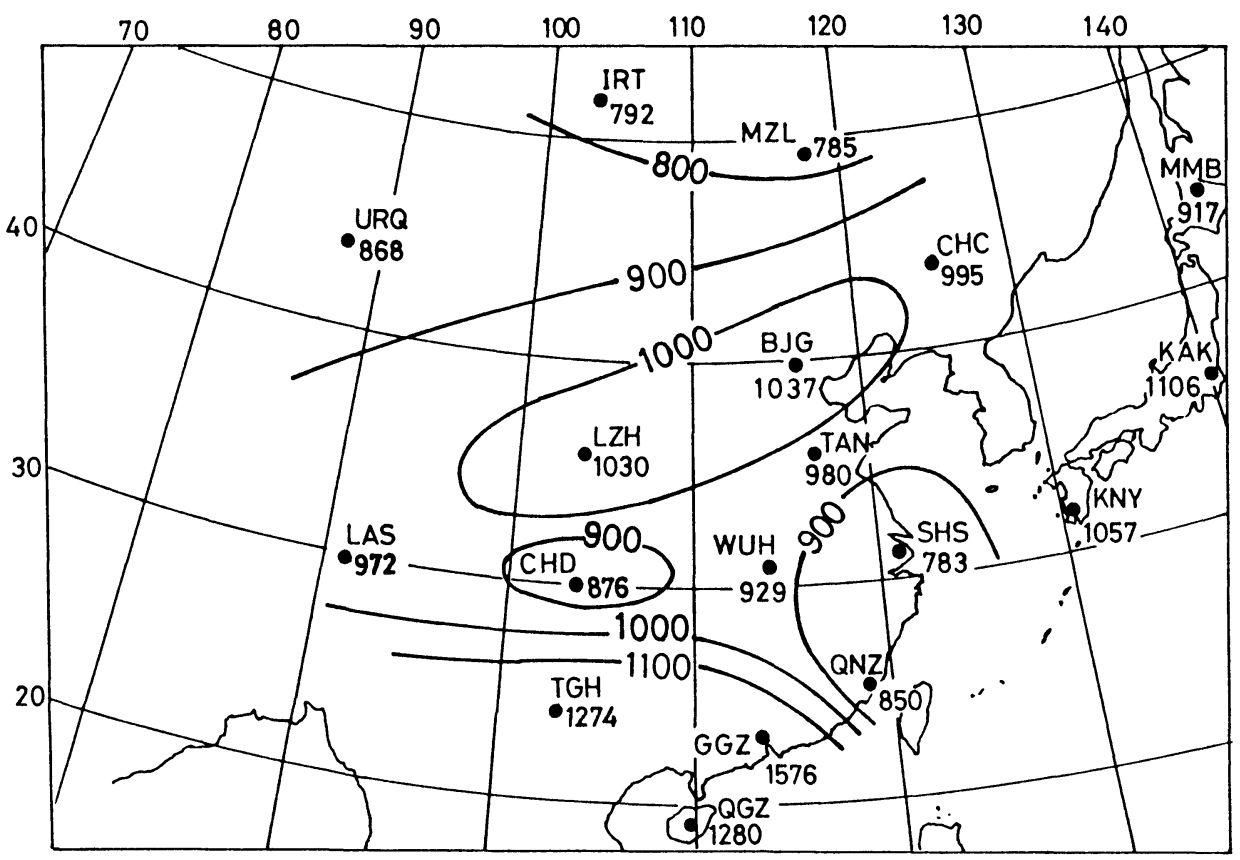

Fig. 1. Contour lines for the apparent depth $h^{*}$ (in units of $\mathrm{km}$ ) of the perfect substitute conductor determined by the 27-day geomagnetic variations in the Chinese region.

perfect substitute conductor in the central and northern Chinese region appears rather small. The average value of $h^{*}$ in this region is about $950 \mathrm{~km}$ and the average value of $\sigma$ is about $1.0 \mathrm{~S} / \mathrm{m}$. However, low conductivity magnitudes and large apparent depth values were found in the southern Chinese region.

SCHULTZ and LARSEN $(1983,1987)$ suggested that it is preferable to use a rotational transformation so that the $D$ magnetic variations are minimized. In this case, the rotated horizontal magnetic components for each observatory might truly satisfy the $P_{1}{ }^{0}$ zonal assumption. The results shown in Figs. 1 and 2 were not obtained through the rotational transformation, because in our practice we found that the possible improvement in the accuracy of $C$ values through the rotational transformation is very limited. To test the validity of our assumption that the rotational transformation is not really necessary in practice in our particular situation, we list in Table 3 two sets of real and imaginary values of $C$ calculated with and without rotation pertaining to the data of several observatories. The rotation angle $\alpha$ is determined from the following formula (SCHULTZ and LARSEN, 1983): 


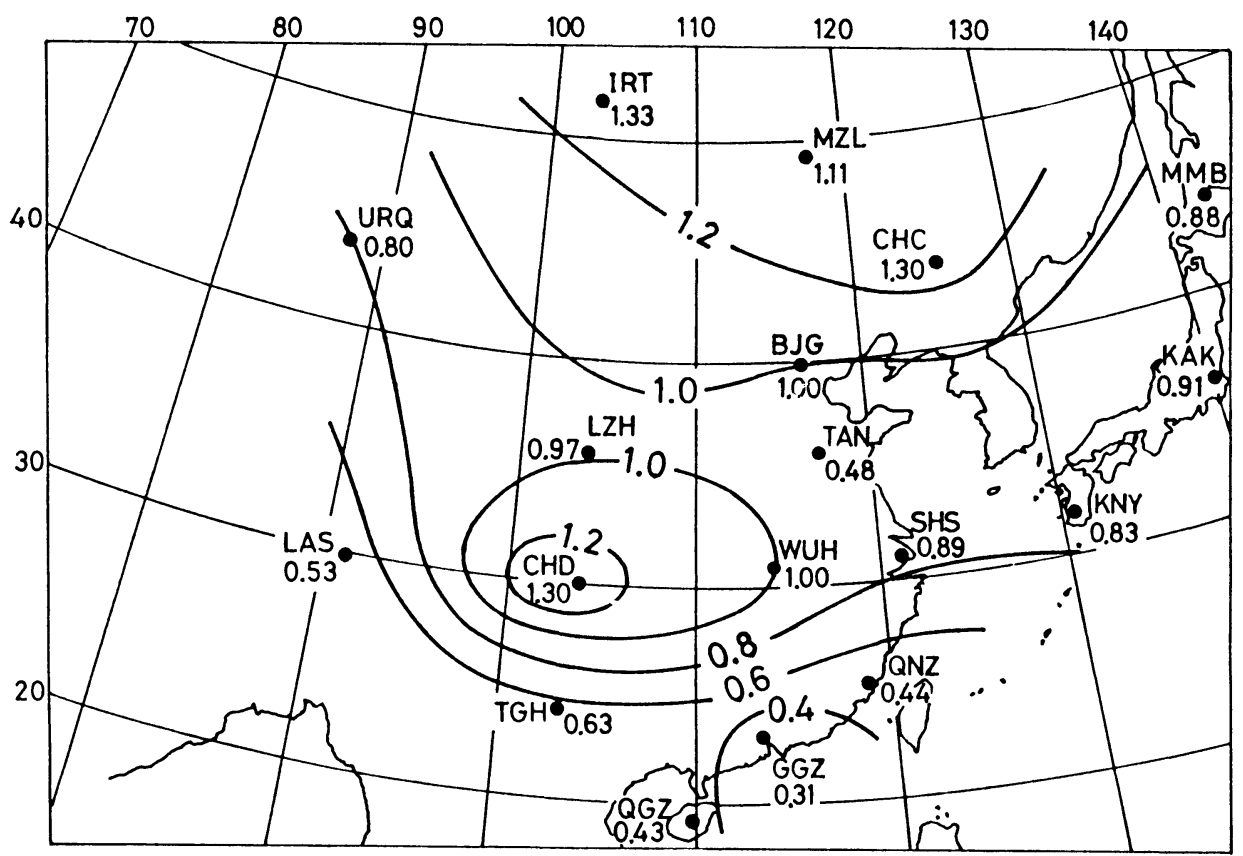

Fig. 2. Contour lines for the electrical conductivity $\sigma$ (in units of $S / m$ ) of the perfect substitute conductor determined by the 27-day geomagnetic variations in the Chinese region.

Table 3. Examination on the possible changes through the rotational transformation.

\begin{tabular}{lcrrc}
\hline \multicolumn{1}{c}{ Site } & Year analysed & \multicolumn{1}{c}{$C$ value $^{(1)}$} & \multicolumn{1}{c}{$C$ value $^{(2)}$} & Rotation angle \\
\hline Wuhan & 1982 & $640.8-230.8 \mathrm{i}$ & $635.5-236.2 \mathrm{i}$ & $+4.00^{\circ}$ \\
Quanzhou & 1983 & $849.6-571.7 \mathrm{i}$ & $843.5-573.7 \mathrm{i}$ & $+1.90^{\circ}$ \\
Guangzhou & 1983 & $1924.5-670.6 \mathrm{i}$ & $1915.8-667.3 \mathrm{i}$ & $+1.26^{\circ}$ \\
Lanzhou & 1984 & $919.6-573.0 \mathrm{i}$ & $919.9-572.4 \mathrm{i}$ & $0.00^{\circ}$ \\
Beijing & 1981 & $1164.0-437.3 \mathrm{i}$ & $1154.4-432.9 \mathrm{i}$ & $-0.44^{\circ}$ \\
Lhasa & 1985 & $972.1-506.2 \mathrm{i}$ & $973.4-505.9 \mathrm{i}$ & $-2.22^{\circ}$ \\
\hline
\end{tabular}

(1) no rotational transformation employed.

(2) $C$ value obtained by using rotational transformation. 


$$
\alpha=\frac{1}{2} \tan ^{-1} \frac{\left\langle Y_{i}(\omega) \cdot X_{i}^{*}(\omega)+Y_{i}^{*}(\omega) \cdot X_{i}(\omega)\right\rangle}{\left\langle\left|X_{i}(\omega)\right|^{2}-\left|Y_{i}(\omega)\right|^{2}\right\rangle},
$$

where $\omega$ is the angular frequency, \langle\rangle denotes the frequency-band-average, $Y_{i}(\omega)$ and $X_{i}(\omega)$ are the east component and the north component respectively and $*$ is the complex conjugate. The frequency band adopted in this study is $(0.0156-0.1953) \mathrm{cpd}$. From Table 3 we can see that the changes for the real part of $C$ are less than $1 \%$, and the changes for the imaginary part of $C$ are less than $2.5 \%$.

\section{Discussion and Conclusion}

1) The Sompi spectral analysis provides more reliable estimates for the response function than the FFT method in the investigation of the long period variation. We stress that the box-car filter used here is not a severe narrow-bandpass filter. In this case, the standard error obtained by the Sompi method is nearly half of the error obtained by the FFT method. This comparison manifests the advantage of the Sompi technique.

2) The lateral change of the deep conductivity structure in the central and northern Chinese region is considered not very large, especially in view of the relatively large error estimates. However, in the southern Chinese region, a relative increase in the apparent depth and a relative decrease in the conductivity are found. We are not sure whether the latter feature is an actual anomalous variation in the deep conductivity structure. Such a feature might be due to the influence of the highly conducting ocean, but the response functions for the Memambetsu, Kakioka, Kanoya and Sheshan do not show this anomalous feature. Besides, the tests on the $C$ values by applying rotational transformation (shown in Table 3) for the data of the Quanzhou and Guangzhou sites, which are located near the ocean, also infer that no anomalous effects due to proximity to ocean are found. Hence, it seems that the influence of the ocean is not an important reason. Secondly, since the southern Chinese observatories are situated in low latitude region, the influence of the equatorial electrojet current might play an important role. Unfortunately, we are not able to present more analyses and discussions in this aspect. Lastly, the poor signal to noise ratio for the $Z$ component in low latitude region might give rise to significant error in the estimation of the response function.

3) Good quality data are obviously very important for the study of long period geomagnetic variation. The qualities of the data in the Lhasa, Guangzhou and Quanzhou observatories seem to be not good enough for this investigation.

We would like to express our thanks to Dr. J. C. Larsen for his valuable comments and suggestions which helped us to refine the original manuscript.

\section{REFERENCES}

Asakawa, E., H. Utada, and T. Yukutake, Application of Sompi spectral analysis to the estimation of the geomagnetic transfer function, J. Geomag. Geoelectr., 40, 447-463, 1988.

BANKS, R. J., Geomagnetic variations and the electrical conductivity of the upper mantle, Geophys. J. R. astr. Soc., 17, 457-487, 1969. 
Hori, S., Y. Fukao, M. Kumazawa, M. Furomoto, and A. Yamamoto, A new method of spectral analysis and its application to the Earth's free oscillations: the "Sompi" method, J. Geophys. Res., 94, 75357553, 1989.

Kumazawa, M., Y. Imanishi, Y. Fukao, M. Furumoto, and A. Yamamoto, A theory of spectral analysis based on the characteristic property of a linear dynamic system, Geophys. J. Int., 101, 613-639, 1990.

Parkinson, W. D. and V. R. S. Hutton, The electrical conductivity of the Earth, in Geomagnetism, Vol. 3, edited by J. A. Jacobs, pp. 261-321, Academic Press, London, 1989.

Pecova, J., K. Pec, and O. Praus, Remarks on spatial distributions of long period variations in the geomagnetic field over European area, in Electromagnetic Induction in the Earth and Moon, edited by U. Schmucker, pp. 171-186, Academic Publications, Japan, 1980.

Roberts, R. G., The long-period electromagnetic response of the Earth, Geophys. J. R. astr. Soc., 78, 547-572, 1984

Roberts, R. G., Global electromagnetic induction, Surv. in Geophys., 8, 339-374, 1986.

Schmucker, U., Anomalies of geomagnetic variations in the south-western United States, Bull. Scripps Inst. Oceanogr., 13, 165 pp., 1970.

Schultz, A. and J. C. LARSEn, Analysis of zonal field morphology and data quality for a global set of magnetic observatory daily mean values, J. Geomag. Geoelectr., 35, 835-846, 1983.

SChultz, A. and J. C. LARSEN, On the electrical conductivity of the mid-mantle-1. Calculation of equivalent scalar magnetotelluric response functions, Geophys. J. R. astr. Soc., 88, 733-761, 1987. 